\title{
L’accord du participe passé dans les SMS francophones du corpus SMS suisse
}

Stark, E ; Riedel, I

DOI: https://doi.org/10.1515/roma.63.5

Posted at the Zurich Open Repository and Archive, University of Zurich

ZORA URL: https://doi.org/10.5167/uzh-88692

Journal Article

Published Version

Originally published at:

Stark, E; Riedel, I (2013). L'accord du participe passé dans les SMS francophones du corpus SMS suisse. Romanistisches Jahrbuch, 63(1):116-138.

DOI: https://doi.org/10.1515/roma.63.5 


\title{
L'accord du participe passé dans les SMS francophones du corpus SMS suisse
}

\author{
Par Elisabeth Stark et Isabelle Riedel*
}

\begin{abstract}
Based on a quantitative empirical analysis of more than 4600 French text messages from the newly established Swiss reference corpus of text messages (cf. www.sms4science.ch), we show that the (graphic) realization of past participle agreement, often considered to be particularly prone to orthographic errors and indicative of more formal registers of contemporary French, is realized in the overwhelming majority of cases in our data $(89,9 \%)$. This holds independently of the sometimes heavily deviant graphic character of the respective text messages and the (very rare) phonic realization of past participle agreement in contemporary French. Significant triggering linguistic factors for the absence of (full) past participle agreement are type and distance of the controller of the agreement (more deviant realizations with relative pronouns than with cliticized objects, more deviant realizations with the controller following the past participle in constructions with être as the auxiliary), and the features of agreement (more deviant realizations of gender than of number features on the past participle), this being in line with important universal typological and theoretical findings. External factors such as sex and education play a certain role, too, which makes past participle agreement in French a sociolinguistic rather than a stylistic variable bound to graphic forms of communication.
\end{abstract}

\section{Introduction}

L'accord du participe passé en français est un chapitre épineux des débats autour de l'orthographe du français, notoirement complexe et difficile à apprendre (cf. Catach 102011, Meisenburg 1996, chap. 3.5). Aussi Marie-José Béguelin pose-telle la question de savoir, dans un article d'orientation plutôt appliquée, s'il faut simplifier les règles d'accord du participe passé (cf. Béguelin 2002, voir plus bas). Cette question se réfère plus précisément à une simplification potentielle du marquage graphique de l'accord du participe passé avec un argument interne du verbe lexical (complément d'objet antéposé ou sujet non-agentif, voir plus bas), étant donné que cet accord se manifeste, depuis environ le $19^{\text {ème }}$ siècle, dans la majorité des cas uniquement à l'écrit, donc dans le code graphique. Il y a rarement, dans le

* Nous tenons à remercier Monique Krötsch, Munich, d'une relecture critique approfondie du texte, qui a permis une amélioration non seulement du niveau de langue. Toutes les erreurs qui restent sont de notre responsabilité. 
code phonique (cf. Hunnius $1980: 247$ s.) $)^{1}$, une réalisation parallèle à l'oral, comme le montrent les exemples suivants :

(1) a. $L a_{\mathrm{FSG}}$ voiture que j'ai acheté-e $e_{\mathrm{FSG}}$ ne me plaît pas.

b. [la F.SG vatyrkəzea $\int t_{\varnothing}$ nəməplepa]

(2) a. La $a_{\mathrm{FSG}}$ lettre que j'ai écrit-e $e_{\mathrm{FSG}}$ n'est pas arrivé-e $e_{\mathrm{F} . S G}$.

b. $\left[\mathrm{la}_{\mathrm{F} . \mathrm{SG}}\right.$ letrəkəzeekri-t $\mathrm{t}_{\mathrm{FSG}} \mathrm{n} \varepsilon$ pazarive $\left._{\varnothing}\right]$

Mis à part quelques participes passés de fréquence élevée, se terminant graphiquement par <-t $>$ ou par $<-s>$ et pouvant réaliser dans le code phonique un accord en [-t] (comme <écrit-e> dans l'exemple 2.b) ${ }^{2}$ ou en [-z] comme marque du féminin (comme $<$ mis-e $>$, le pluriel n'étant jamais exprimé dans le code phonique), la majorité des participes passés du français moderne ne réalisent pas phoniquement l'accord en genre avec leurs arguments respectifs (comme <acheté-e> ou $<$ arrivé-e> dans les exemples 1.b et 2.b) :

Im Laufe des 18. Jahrhunderts vollzieht sich ein entscheidender Wandel. Das accordProblem büßt als sprachliches Problem ein Großteil seiner Bedeutung ein. Die bis dahin lautlich geschiedenen maskulinen und femininen Partizipialformen fallen zusammen, da die auf Vokalquantität beruhende Opposition neutralisiert wird. Die Bedeutung des accord-Phänomens verlagert sich in großem Umfang von der Sprache auf die Graphie, da die Schreibung - wie üblich - dem Wandel keine Rechnung trägt und die Entwicklung zur Invarianz nicht mitvollzieht. (Hunnius 1980 : 247 s.)

Pour concrétiser un peu les dimensions du phénomène dans l'usage du français contemporain, citons quelques chiffres : dans son analyse de 8000 verbes du français contemporain, Tanase 1976 conclut que seulement 1,25\% de ces verbes (types) marquent phoniquement le genre dans l'accord du participe passé. Hunnius 1980 souligne pourtant qu'il faut aussi considérer la fréquence de ces participes (tokens), et estime que, en vertu de la fréquence élevée de certains d'entre eux dans l'usage, $10 \%$ de tous les tokens (des participes passés) attestent un marquage " audible » (de l'accord en genre avec leur argument) (cf. Hunnius 1980 : 249 s., voir aussi Gutwin 1996 : 9 s.). Cela signifie que dans la plupart des cas, c'est-à-dire dans $90 \%$ des occurrences des participes passés en français, l'accord en genre n'est pas audible.

A tout cela s'ajoute une observation assez répandue dans la discussion linguistique d'orientation variationniste, à savoir le constat que l'absence d'accord du

1 On l'inclut pour cette raison dans les phénomènes dits d' « orthographe grammaticale » (cf. Catach ${ }^{10} 2011$ : 45, Meisenburg 1996 : 197), c'est-à-dire en tant que marquage exclusivement graphique d'informations morphosyntaxiques dans la phrase du français moderne, comme p. ex. le nombre et la personne du verbe conjugué en accord avec le sujet dans la plupart des formes verbales (cf. Meisenburg 1996 : 197-200).

2 Pour les détails des régularités graphématiques du français qui en sont responsables voir Meisenburg 1996 : 189-200. En règle générale, une syllabe graphique ouverte qui consiste en une seule lettre : <-e>, après consonne graphique, comme dans <écrit-e>, indique la réalisation phonique de cette consonne. 
participe passé, là même où il serait phoniquement possible, serait un trait du " français de l'immédiat » (cf. Koch / Oesterreicher 22011 : 169, Söll / Hausmann 1985 : 115 ss.), donc d'une variété du français qui se caractérise par un certain degré d'informalité de la situation de communication respective (cf. Koch / Oesterreicher 2001 : 586). Cette opinion se reflète aussi dans la citation suivante, prise à titre illustratif à la nouvelle édition de la grammaire de référence de Riegel / Pellat / Rioul (72009) : " La tendance actuelle est à l'invariabilité du participe non seulement à l'oral, où une phrase comme Quelle impression vous a-t-il produite? sonne bizarre, mais aussi chez les meilleurs auteurs modernes » (Riegel / Pellat / Rioul ${ }^{7} 2009$ : 503).

Béguelin 2002, qui observe également cette tendance, va un pas plus loin et propose, en conséquence de cette évolution, une simplification des règles de l'accord du participe passé. Elle fonde cette suggestion sur des observations faites sur des élèves ayant des difficultés à appliquer graphiquement l'accord du participe passé. De plus, elle constate une perte d'importance " socio-indexicale » de l'accord du participe passé dans les communautés francophones, phénomène qui a assumé longtemps la fonction de « signal élitiste », p. ex. dans les concours de dictée, et semble perdre cette fonction surtout avec l'expansion rapide de la communication médiée par ordinateur (CMO), dont les SMS font partie (cf. Panckhurst $2006: 345)$ :

À quoi bon inculquer un catéchisme officiel indigeste, truffé d'exceptions et de cas particuliers, si l'on peut démontrer qu'il n'est qu'un montage aléatoire, fait d'additions successives dont le bien-fondé linguistique et la cohérence sont douteux ? Comme, de surcroît, le type d'apprentissage en cause semble avoir perdu une bonne part de la pertinence sociale qu'il pouvait avoir avant l'ère de la communication électronique, ne conviendrait-il pas de revoir, sur cette question, à la fois la doctrine et les contenus d'enseignement? (Béguelin $2002:$ 165)

Ces réflexions souffrent cependant toutes d'un manque d'évidence empirique regrettable, surtout d'analyses quantitatives basées sur un nombre suffisamment important d'exemples provenant de corpus authentiques et comparables entre eux (cf. les remarques à ce sujet de Söll / Hausmann 1985 : 117). Même si l'absence d'accords « audibles » non réalisés, notée ça et là dans les descriptions, est un fait, sa fréquence et son analyse linguistique morphosyntaxique restent jusqu'à nos jours un desideratum de la linguistique variationniste du français. En d'autres termes, nous ne savons toujours pas si nous avons affaire à de simples " erreurs » dues, entre autres, aux conditions de production du discours plus ou moins spontané (effets de performance, « on line-syntax », cf. Auer 2000), ou si l'absence d'accord du participe se montre, dans certaines formes de communication avec une fréquence et une régularité suffisantes pour pouvoir être considérée comme marqueur ou indicateur variationnel (cf. Halliday 1978, Dufter / Stark 2002 : 94). C'est à cette question que se consacrera la présente contribution.

Avant de présenter la base empirique de nos observations (chapitre 3), nous résumerons dans le chapitre 2 les régularités morphosyntaxiques sous-jacentes à l'accord du participe passé en français standard contemporain pour en dé- 
duire nos paramètres d'analyse. Le chapitre 4 présentera les résultats quantitatifs de notre étude, tandis que le chapitre 5 tentera de donner une interprétation aux phénomènes identifiés. L'article se terminera sur une brève conclusion (chapitre 6).

\section{L'accord du participe passé en français standard contemporain}

En général, le phénomène de l'accord en linguistique peut être défini comme suit : " The term agreement commonly refers to some systematic covariance between a semantic or formal property of one element and a formal property of another » (Steele $1978: 10)$.

D'après Corbett 2006, la notion essentielle dans cette citation est celle de covariance, qui spécifie le fait que lorsqu'un élément change de forme, un autre va aussi changer de forme (cf. Corbett $2006: 4$ ).

Corbett 2006 identifie quatre éléments centraux pour que l'accord puisse se faire. Nous les utiliserons par la suite pour notre analyse de l'accord du participe passé dans les SMS. Le domaine constitue la configuration syntaxique dans laquelle l'accord se produit. Le contrôleur est l'élément qui détermine l'accord par ses traits inhérents et le déclenche pour ainsi dire. L'élément qui change de forme et est marqué selon les valeurs des traits du contrôleur est la cible. Les traits représentent les propriétés morphosyntaxiques concernées par et exprimées dans l'accord (cf. Corbett $2006: 4$ s.).

Nous décrirons dans ce qui suit les facteurs qui déclenchent l'accord du participe passé en français standard contemporain. Rappelons brièvement pour ce faire le développement et la présence de l'accord du participe passé en français et dans les autres langues romanes.

Le latin connaissait deux périphrases verbales perfectives, à savoir les constructions avec esse plus participe passé, et les constructions avec habere plus participe passé (cf. Loporcaro 1998 : 7). Ces deux types se sont maintenus dans la majorité des langues romanes. Il y a des langues et des variétés romanes qui connaissent les deux types, et ont donc une alternance d'auxiliaire pour les constructions perfectives (comme le français, l'italien, l'occitan et le piémontais), mais il y a aussi des langues et variétés romanes qui ne connaissent qu'un seul type, et par conséquent n'ont pas d'alternance d'auxiliaire (comme l'espagnol, le portugais, le roumain et aussi le wallon ; cf. Lois 1990 : 234). L'espagnol, le portugais et le roumain forment leurs temps perfectifs avec l'équivalent de avoir, et ne font l'accord du participe passé que dans les constructions passives, qui se forment avec un équivalent d'être (cf. Loporcaro 1998 : 9 s.). Selon Loporcaro, deux paramètres fondamentaux déterminent l'accord du participe passé dans les langues romanes : la sélection du verbe auxiliaire et, dans le cas des verbes transitifs, l'ordre linéaire entre participe passé et objet direct. En outre, si l'accord du participe passé avec le sujet est réalisé, c'est l'auxiliaire être qui est utilisé.

En général, l'accord entre le contrôleur et le participe passé se réalise en français contemporain avec les deux auxiliaires avoir et être. Il se produit également sans verbe auxiliaire. Ces trois domaines différents sont à distinguer. 
Quand le participe passé a la valeur d'un adjectif (cf. Riegel / Pellat / Rioul 72009 : 502) et se trouve en position adnominale, il apparaît comme ajout au substantif, sans verbe auxiliaire ni copule (cf. Gutwin 1996 : 11). Il s'accorde en genre et en nombre avec le substantif qu'il modifie comme tout adjectif adnominal :

(3) les $_{(\mathrm{F}) \mathrm{PL}}$ illusions perdu-e-s-PL (Riegel / Pellat / Rioul 72009 : 502)

Même chose pour le participe passé comme complément prédicatif dans les constructions avec copule.

L'auxiliaire être s'utilise également dans les constructions inaccusatives, passives et réfléchies. Dans toutes les constructions avec être comme auxiliaire ou copule, le participe passé s'accorde avec le sujet de la phrase ou avec le clitique réfléchi coréférentiel (sauf dans les constructions réfléchies si le clitique réfléchi est complément d'objet indirect, cf. Jones 1996 : 93), indépendamment de la position pré- ou postverbale du sujet :

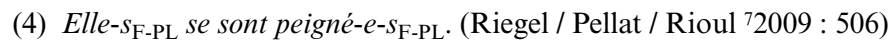

Le clitique réfléchi se correspond, dans cet exemple et dans les constructions qui lui sont comparables, à un complément d'objet direct, ce qui permet de faire l'accord avec le réfléchi antéposé au participe passé et coréférentiel au sujet (cf. Riegel / Pellat / Rioul 72009 : 506). Définissons ensuite les constructions dites inaccusatives, qui sont structurellement très similaires aux constructions passives et sélectionnent comme celles-ci l'auxiliaire être, ce qui conséquemment déclenche l'accord du participe passé avec le sujet de la phrase :

Intransitive (ergative) Verben wie arriver [...] sind einstellige Prädikate, sie werden auch als unakkusative Verben bezeichnet. Bei den [unakkusativen] Verben geht man davon aus, dass das einzige Argument auf einer zugrundeliegenden Strukturebene ein direktes Objekt (direktes internes Argument) ist, kein Subjekt (externes Argument), und somit in der kanonischen Objektposition generiert und später in die Subjektposition verschoben wird. Die Subjekte von [unakkusativen] Verben sind somit vergleichbar mit den Subjekten in Passivsätzen, welche zugrundeliegende Objekte darstellen. (Müller $2000: 1 \mathrm{~s}$.)

L'exemple suivant, avec le verbe de mouvement arriver, illustre bien la construction inaccusative :

(5) $\operatorname{Les}_{(\mathrm{F}) \mathrm{PL}}$ filles sont arrivé-e-s-s-PL. (Jones $1996: 93$ )

Dans toutes ces constructions, mais surtout dans les constructions inaccusatives, le sujet peut être aussi postposé au verbe conjugué et au participe passé ; l'accord, aussi celui en personne et nombre entre le sujet et le verbe conjugué, peut être défectif dans certaines variétés dans ces configurations syntaxiques, comme le montre l'exemple suivant en français soutenu (cf. Remberger / Mensching 2006 : 177) :

(6) Il est $_{\mathrm{SG}}$ arrivé $_{(\mathrm{M} .) \mathrm{SG}}$ trois fille- $s_{(\mathrm{F}) \mathrm{PL}}$.

Cependant, un sujet antéposé au verbe conjugué doit s'accorder en nombre avec celui-ci, sinon la dérivation est agrammaticale, dans toutes les variétés, comme on le voit dans l'exemple (7) :

(7) * Trois $_{\mathrm{PL}}$ fille-s $_{(\mathrm{F} .) \mathrm{PL}} e t_{\mathrm{SG}}$ arrivé $_{(\mathrm{M} .) \mathrm{SG}}$. 
Cette régularité, découverte par Joseph Greenberg, est formulée dans son fameux universel 33 : "When number agreement between the noun and the verb is suspended and the rule is based on order, the case is always one in which the verb precedes and the verb is in the singular » (Greenberg 1963 : 74). Nous pourrions en déduire que l'ordre relatif entre le contrôleur et la cible joue aussi un rôle pour l'accord du participe passé avec l'auxiliaire être (ce qui est toujours le cas pour l'auxiliaire avoir, dont il sera question plus loin) : il pourrait être défectif dans certaines variétés, quand le sujet est postposé.

Dans les constructions avec l'auxiliaire avoir, le participe passé s'accorde avec un complément d'objet direct qui lui est antéposé. Nous allons examiner dans quels domaines syntaxiques et avec quelles catégories grammaticales de l'objet direct cela est possible. Le cas le plus fréquent est certainement celui des objets clitisés toujours antéposés au participe passé (ces éléments-là ne se trouvent donc jamais dans la position canonique originale des compléments d'objet directs) :

(8) A. Qu'as tu fait de ce-tte F.SG $_{\text {lettre }}(\mathrm{F}) ?^{3}$

B. Je $l_{\mathrm{FSG}}$ 'ai remis- $e_{\mathrm{F} . S G}$ à qui de droit.

Le clitique la dans la réplique de $\mathrm{B}$ reprend l'objet direct de la phrase précédente cette lettre. Le clitique assume le rôle du contrôleur, qui transmet au participe passé, la cible, les valeurs [féminin] pour le trait d'accord [genre], et [singulier] pour le trait d'accord [nombre].

Il y a aussi accord quand l'objet direct est un pronom relatif antéposé au verbe :

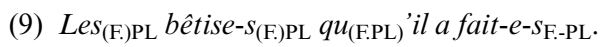

Dans ce cas, le participe passé s'accorde avec l'antécédent du pronom relatif que ou, plus précisément, aux traits hérités de celui-là. Il est à noter que, dans cette construction, la distance structurelle entre le contrôleur et la cible est très grande, du fait que l'objet direct a quitté sa place canonique postverbale et a été même antéposé au sujet de la phrase relative, dans une position encore plus lointaine de sa position originale que dans le cas des objets clitisés. L'accord peut se faire aussi avec d'autres types de déplacements $q u$ - (cf. p. ex. Laenzlinger 2003, chap. 6). Dans l'exemple suivant, il s'agit d'un mot interrogatif (combien, qui fait sémantiquement et fonctionnellement partie des "éléments $q u$ - " malgré sa forme) introduisant l'objet direct antéposé à la phrase interrogative entière et déclenchant par cette position l'accord avec le participe passé :

\section{(10) Combien de femme-s $s_{(\mathrm{F}) \mathrm{PL}}$ Don Juan a-t-il séduit-e- $s_{\mathrm{F}-\mathrm{PL}}$ ?}

L'accord enfin se réalise aussi quand un syntagme nominal, également quantifié, ou un pronom en fonction d'objet direct est antéposé au participe passé, par exemple dans une construction clivée :

(11) C'est tout- $e_{\mathrm{F}}$ un- $e_{\mathrm{FSG}}$ ville $_{\mathrm{F})}$ que le séisme a détruit- $e_{\mathrm{FSG}}$.

\footnotetext{
3 Tous les exemples suivants de ce chapitre sont tirés de Riegel / Pellat / Rioul 72009 : $502 \mathrm{~s}$.
} 
L'objet direct toute une ville, extrait de la phrase Le séisme a détruit toute une ville, se trouve focalisé dans une position précédant le participe passé, d'où la possibilité de faire l'accord.

Nous constatons que le complément d'objet direct précédant le participe passé dans les constructions avec avoir peut être aussi bien un syntagme nominal lexical (y compris les noms propres) qu'un pronom personnel, un clitique (aussi réfléchi), un pronom relatif ou interrogatif («élément $q u-»)$. S'il y a donc, avant le participe passé, un élément qui a la fonction complément d'objet direct (cod) sans en occuper la position canonique postverbale, ce dernier déclenche automatiquement l'accord du participe passé (cf. Gutwin 1996 : 109 s.).

Sur la base de ces descriptions structurales des régularités de l'accord du participe passé (qui font abstraction de certains cas spéciaux dont témoignent notamment Riegel / Pellat / Rioul 72009 : 503 ss. et qui ne seront pas pris en compte en détail dans ce qui suit), il est facile de formuler désormais avec Loporcaro 1998 la règle générale sous-jacente à tous les cas d'accord du participe passé en français ${ }^{4}$. Assumons que $b$ est une proposition, $a$ un nominal de $b$, et $p$ un participe passé d'une forme verbale périphrastique de $b . P$ s'accorde en genre et en nombre avec $a$, si :

i) La proposition est finalement intransitive (= il n'y a pas de complément d'objet direct dans sa position canonique postverbale).

ii) $a$ est autorisé au contrôle de l'accord. Un nominal est autorisé au contrôle de l'accord si :

a. il n'est pas chômeur = il se trouve dans une position argumentale ;

b. il est le 2-initial de $p=$ il est le complément d'objet direct original du verbe lexical sur lequel est formé le participe passé en question (cf. Loporcaro $1998: 53)$.

Cette formule permet d'expliquer tous les cas d'accord du participe passé en français standard contemporain ${ }^{5}$. En outre, elle contient déjà les deux facteurs

4 Loporcaro 1998 formule ces régularités dans la terminologie et la perspective de la grammaire relationnelle, suivant Perlmutter 1978. Nous essayons de faire abstraction de l'appareil théorique, tout en gardant les précieuses généralisations de Loporcaro et en paraphrasant ses formulations de manière plus traditionnelle et plus conforme avec les observations discutées auparavant.

5 Aussi et surtout les cas qui sont traditionnellement considérés et énumérés (Riegel / Pellat / Rioul 72009 : 503 ss.), comme des « exceptions » ou des cas spéciaux, comme p. ex. les compléments quantitatifs de verbes comme mesurer ou peser, qui ne sont pourtant pas compléments d'objet direct, ni sémantiquement, ni syntaxiquement (ce que démontre le test de substitution par des éléments interrogatifs en combien, et non pas en que; l'impossibilité de représenter le sujet d'une phrase au passif etc.) et ne peuvent donc pas déclencher l'accord, ceci en désaccord avec Béguelin 2002 : 165, qui considère problématique et floue la notion de complément d'objet direct, ce qui n'est pas le cas, du moins pas au niveau syntaxique. 
linguistiques les plus importants, déclenchant ou inhibant l'accord du participe passé : d'abord, la construction en question (prédicative, inaccusative, passive, réfléchie ou transitive, mais sans complément d'objet direct à sa place canonique) ; ensuite, l'ordre des éléments en question (argument interne / complément d'objet direct et prédicat verbal ou adjectival), c'est-à-dire, la position du complément d'objet direct initial préverbale ou en position structurale de sujet de la phrase. Ce sont ces deux facteurs linguistiques principaux, avec des sous-catégories là où c'est nécessaire, qui guideront l'analyse empirique présentée dans le chapitre 4. Avant de procéder à cette analyse, nous aimerions présenter brièvement notre base de données, à savoir le corpus sms4science.ch.

\section{Base de données et facteurs d'analyse}

Dans ce qui suit, nous présentons brièvement le projet sms4science.ch dont sont issues nos données. Nous montrerons ensuite comment nous avons procédé dans l'analyse du corpus.

En automne / hiver 2009 / 2010, les universités de Zurich et de Neuchâtel ont recueilli des SMS authentiques de toute la Suisse, et en mai / juin 2011, un appel ciblé a été lancé en collaboration avec l'université de Berne pour recueillir les variétés italiennes et romanches-grisonnes. Cette action a été soutenue par les médias et la Swisscom, la compagnie téléphonique la plus importante de la Suisse. Les utilisateurs de téléphones mobiles ont été invités à transmettre, à un numéro gratuit, une copie de chaque SMS qu'ils envoyaient et à remplir un questionnaire sociolinguistique anonyme sur internet. Les données ainsi rassemblées ont ensuite été intégrées au corpus sms4science.ch. En remplissant le questionnaire, les participants ont donné leur accord pour l'utilisation de leurs réponses dans le cadre de différents projets scientifiques lancés par les deux universités. Les SMS et les réponses au questionnaire ont été enregistrés dans une banque de données, dans le but d'étudier le phénomène de la communication par SMS en Suisse (cf. http:// www.sms4science.ch/). 25.947 SMS ont été ainsi collectionnés, dont $18 \%$ en français ou variété du français. Ces SMS ont été envoyés par 2.784 personnes. Parmi les participants, 1.316 ont rempli le questionnaire anonyme concernant les facteurs socio-démographiques (domicile, âge, sexe, langue maternelle, éducation etc.). 909 personnes ont déclaré avoir un dialecte suisse allemand comme langue maternelle, 165 ont indiqué l'allemand, 257 le français, 107 l'italien et 64 le rhéto-roman. 129 personnes ont nommé une autre langue comme langue maternelle (plusieurs réponses étaient possibles). $46 \%$ des participants étaient âgés de 20 à 29 ans, mais il faut mentionner que 40 personnes de plus de 60 ans ont envoyé leurs SMS et rempli le questionnaire.

Le corpus sms4science.ch montre une forte tendance à l'alternance codique. En outre, il montre une préférence pour les dialectes (surtout dans les SMS en allemand, romanche et italien). Au total, le corpus englobe plus de 20 langues et variétés diverses (entre autres l'allemand, le français, l'italien, l'anglais, l'espagnol, le néerlandais, le suédois et le portugais). 10.718 SMS (environ 41\%) sont rédigés en un dialecte suisse alémanique, 7.224 SMS sont en allemand non-dialectal et 
4.627 (SMS) en français (dont 26 en un patois du français) ${ }^{6}$. Ces langues et variétés sont donc les plus fréquemment représentées dans le corpus.

Notre analyse se concentre sur les SMS en français. Nous avons retenu par analyse manuelle tous ceux contenant un accord du participe passé. Il faut souligner que ces SMS viennent presque exclusivement de locuteurs natifs du français, ce qui sera important au vu des résultats.

Les facteurs suivants à analyser ont été définis et étudiés séparément pour chaque auxiliaire, avoir et être.

Comme premier facteur, nous avons considéré la catégorie grammaticale du contrôleur. Les contrôleurs avec lesquels l'accord peut se réaliser sont les suivants : pronom / pronom clitique (y compris les sujets clitiques), DP (y compris les noms propres), objet clitisé, pronom relatif ou élément $q u$-. Comme deuxième facteur, et uniquement pour l'auxiliaire être, nous avons analysé de plus près le domaine de l'accord, plus précisément nous nous sommes posé la question de savoir si le contrôleur de l'accord du participe avec l'auxiliaire être, le sujet, est en position pré- ou postverbale. De plus, les différentes constructions avec cet auxiliaire ont été prises en considération : le passif, une construction inaccusative, réflexive ou prédicative (cf. chapitre 2). Comme troisième facteur ont été analysés les traits de l'accord, le genre et le nombre, et pour chaque valeur des traits, la réalisation ou non de leur marquage sur le participe passé en question. Pour chaque participe, nous nous sommes demandé quelle combinaison des traits [masculin, M] ou [féminin, F], [singulier, $\mathrm{Sg}$ ] ou [pluriel, $\mathrm{Pl}$ ], avait été réalisée. Cette approche nous permet d'examiner si l'accord est marqué majoritairement pour le genre ou pour le nombre, et quelle valeur, quelle combinaison des traits ont provoqué davantage d'accords non-marqués.

En ce qui concerne la cible, à savoir le participe passé, le marquage de l'accord en genre, audible ou non audible, a été analysé comme quatrième facteur. Ceci dans l'intention de déceler si l'audibilité de l'accord a une influence sur sa réalisation graphique correcte.

Enfin, nous avons également pris en considération dans notre analyse les facteurs extralinguistiques. Il s'agit des facteurs suivants : sexe, âge et éducation. Nous voulions savoir si ceux-ci ont une influence sur le marquage de l'accord.

Les résultats en chiffres bruts de l'analyse ont été ensuite analysés avec le programme statistique SPSS, pour identifier la pertinence de certains facteurs ou certains phénomènes présents dans le corpus suisse de SMS en français.

\footnotetext{
${ }^{6}$ Contrairement aux chiffres présentés dans ce qui suit, le nombre actuel de SMS en français dans notre corpus s'est vu réduire de deux (4.627 au lieu de 4.629), du fait d'une correction de l'étiquettage pour les langues après que nous avions fait nos analyses. Cette différence ne change pourtant en rien les résultats statistiques généraux, et ne sera donc plus prise en compte par la suite.
} 


\section{Résultats de l'analyse de corpus}

L'analyse manuelle des 4.629 SMS en français du corpus suisse a identifié 710 participes passés susceptibles d'accord avec un argument interne de la construction, dont 107 cas avec avoir, 409 avec être et 194 constructions elliptiques avec être. Dans les ellipses, le sujet, l'auxiliaire ou les deux peuvent être omis. Il s'est avéré que le sujet est omis dans $86,1 \%$ de toutes les constructions elliptiques. Dans 13,9\%, l'auxiliaire est omis. Voir les deux exemples ci-dessous :

(12) Coucou o suis bien arrivé chez GM. [...].

(13) Montre o retrouvée là où elle l'avait laissée. [...].

\subsection{Résultats généraux}

Le tableau 1 montre la fréquence avec laquelle est marqué l'accord du participe passé dans l'ensemble du corpus :

\begin{tabular}{|l|l|l|l|l|}
\hline & $\begin{array}{l}\text { Chiffres } \\
\text { absolus }\end{array}$ & $\begin{array}{l}\text { Pourcen- } \\
\text { tage }\end{array}$ & $\begin{array}{l}\text { Pourcentage } \\
\text { valable }\end{array}$ & $\begin{array}{l}\text { Pourcentage } \\
\text { accumulé7 }\end{array}$ \\
\hline Non marqué & 72 & 10,1 & 10,1 & 10,1 \\
\hline Marqué & 638 & 89,9 & 89,9 & 100,0 \\
\hline Total & 710 & 100,0 & 100,0 & \\
\hline
\end{tabular}

Tableau 1 : Marquage de l'accord du participe passé dans le corpus entier

« Marqué » veut dire dans ce contexte que l'accord du participe passé correspond aux régularités du français standard (et de son orthographe). "Non-marqué » veut dire qu'il y a déviance par rapport à ces régularités : soit absence de l'accord tout court comme dans le deuxième participe passé de l'exemple (14) cf. infra, soit marquage partiel, ou marquage non correspondant aux traits du contrôleur, comme dans l'exemple (15):

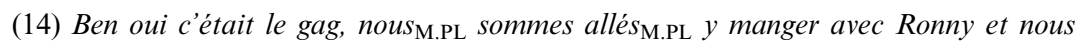
n'avons pas pu résister à prendre les ravioli Carly quand nous les $s_{\mathrm{M} . \mathrm{PL}}$ avons $v u_{\mathrm{M} . \mathrm{SG}}$ sur la carte.

(15) Coucou Evelyne! La $a_{\mathrm{FSG}}$ route était bloqué(M.)SG à Lugano. Nous avons donc perdu une demi heure. Je pense que nous arrivons à 14h30. Je t'appelle quand nous sommes à Bienne.

En règle générale, l'accord du participe passé est marqué dans notre corpus dans presque $90 \%$ des cas possibles de façon « correcte », chiffre assez élevé vus

7 La colonne « Pourcentage accumulé » additionne les résultats de la colonne précédente «Pourcentage valable». Progressivement, un résultat est ajouté au résultat précédent. Ainsi la colonne « Pourcentage accumulé » représente-t-elle les résultats de ces additions. 
les constats mentionnés dans le chapitre 1 sur la perte ou l'absence d'accord dans le « français de l'immédiat » dont relèvent, sans aucun doute, nos SMS (cf. Stark 2011 : 17). Il se peut donc que le médium graphique favorise le maintien du marquage de l'accord aussi dans la communication informelle, ce qui reste à démontrer dans des corpus oraux par des études quantitatives fiables.

Quant aux déviances de l'accord par rapport aux régularités du français standard contemporain, c'est l'auxiliaire avoir qui les déclenche le plus fréquemment, comme on le voit dans le tableau 2 :

\begin{tabular}{|l|l|l|l|}
\hline & Non marqué & Marqué & Total \\
\hline Avoir & 25 & 82 & 107 \\
& $23,4 \%$ & $76,6 \%$ & $100,0 \%$ \\
\hline Etre & 32 & 377 & 409 \\
& $7,8 \%$ & $92,2 \%$ & $100,0 \%$ \\
\hline Etre ellipses & 15 & 179 & 194 \\
& $7,7 \%$ & $92,3 \%$ & $100,0 \%$ \\
\hline Total & 72 & 638 & 710 \\
& $10,1 \%$ & $89,9 \%$ & $100,0 \%$ \\
\hline
\end{tabular}

Tableau 2 : Marquage de l'accord du participe passé en fonction de l'auxiliaire

25 participes passés avec avoir ne sont pas accordés à leur contrôleur (donc $23,4 \%$ des cas), tandis qu'un accord incorrect ou incomplet se trouve dans seulement 7,8\% des cas avec être, et dans 7,7\% des cas avec être en construction elliptique. Ce résultat n'est pas surprenant. Rappelons que l'accord se fait dans le premier cas avec un objet direct préposé au participe et non pas avec un sujet en position préverbale, qui reste dans sa position canonique. La structure syntaxique est donc plus complexe. Regardons, pour illustrer ce fait, l'exemple suivant :

(16) Ouais c'est plutôt salope ou sale connasse. Tu c'est quoi à manor j'ai trouvé la pre-

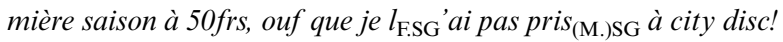

Ici, le participe passé " pris » est dans la forme par défaut, qui correspond à celle du masculin singulier, sans marque aucune de la valeur [féminin] du trait [genre] du contrôleur précédent la.

\subsection{Le rôle du domaine et du contrôleur}

Dans ce sous-chapitre, nous nous demandons si le domaine et le contrôleur ont une influence sur le marquage de l'accord.

\subsubsection{Avoir}

Le tableau suivant montre que le contrôleur de l'accord du participe passé avec avoir est dans la majorité des cas, c'est-à-dire $80,4 \%$, un objet clitisé. Au deuxième rang figure, mais uniquement avec $18,7 \%$, le pronom relatif, un élément $q u$-. Il est 
frappant de constater que le contrôleur n'est jamais une DP. De plus, le contrôleur est seulement une fois un élément interrogatif ( $q u-)$.

\begin{tabular}{|l|c|l|l|l|}
\hline & $\begin{array}{l}\text { Chiffres } \\
\text { absolus }\end{array}$ & $\begin{array}{l}\text { Pourcen- } \\
\text { tage }\end{array}$ & $\begin{array}{l}\text { Pourcentage } \\
\text { valable }\end{array}$ & $\begin{array}{l}\text { Pourcentage } \\
\text { accumulé }\end{array}$ \\
\hline Objet clitisé & 86 & 80,4 & 80,4 & 80,4 \\
\hline Pronom relatif $(q u-)$ & 20 & 18,7 & 18,7 & 99,1 \\
\hline Elément interrogatif $(q u-)$ & 1 & 0,9 & 0,9 & 100,0 \\
\hline Total & 107 & 100,0 & 100,0 & \\
\hline
\end{tabular}

Tableau 3 : Contrôleurs de l'accord avec avoir

Bien que le nombre des différents cas soit trop insignifiant pour permettre des déclarations bien fondées, on peut voir dans le tableau 4 et l'exemple (17) plus bas que c'est principalement le pronom relatif, suivi d'objets clitisés, en tant que contrôleur de l'accord qui, dans environ un tiers des cas, déclenche un non-accord :

\begin{tabular}{|l|l|l|l|}
\hline & Non marqué & Marqué & Total \\
\hline Objet clitisé & 19 & 67 & 86 \\
& $22,1 \%$ & $77,9 \%$ & $100,0 \%$ \\
\hline Pronom relatif $(q u-)$ & 6 & 14 & 20 \\
& $30,0 \%$ & $70,0 \%$ & $100,0 \%$ \\
\hline Elément interrogatif $(q u-)$ & 0 & 1 & 1 \\
& $0,0 \%$ & $100,0 \%$ & $100,0 \%$ \\
\hline Total & 25 & 82 & 107 \\
& $23,4 \%$ & $76,6 \%$ & $100,0 \%$ \\
\hline
\end{tabular}

Tableau 4 : Marquage de l'accord en fonction de la catégorie du contrôleur de l'accord avec avoir

(17) Hi! Tu t'rappel d'la commande pr Matthew Rocher $k_{\mathrm{F} . \mathrm{SG} .}$ on avait fait ${ }_{(\mathrm{M} .) \mathrm{SG}} a c$ maman? Tu $l_{\mathrm{FSG} .}$ 'avais mise ${ }_{\mathrm{FSG}}$ à la poste? [...].

Tous les éléments coréférentiels avec la commande dans cet exemple, le pronom relatif que (ici dans sa variante d' « orthographe phonographique » $<\mathrm{k}>$, cf. Anis 2007 : 97) et le clitique la, portent la valeur [féminin] du trait [genre], mais cette valeur est marquée une fois seulement sur la cible $(<$ mise $>)$ après l'objet clitisé, pas sur $<$ fait $>$, contrôlé par le pronom relatif. Cette variation à l'intérieur d'un même SMS indique une variation intra-individuelle importante, probablement déclenchée par des facteurs structuraux (voir infra, chapitre 5) et rend peu plausibles les explications basées sur la variation intra-individuelle, déclenchée par des facteurs externes (variation diaphasique, variation entre « immédiat et distance communicative $»)$. 


\subsubsection{Etre}

Il s'est avéré, dans l'analyse du corpus (cf. Riedel 2012 : chap. 7.2.2), que le type de construction avec être, c'est-à-dire les constructions passives, inaccusatives, réfléchies et prédicatives, n'a pas d'impact sur le marquage de l'accord, et pas davantage dans les structures elliptiques avec être (comme dans l'exemple (4) cité plus haut, avec une construction passive elliptique).

Cela vaut aussi pour la catégorie du contrôleur ; nous n'avons pas pu observer de différences quant à la réalisation de l'accord entre des sujets pronominaux / clitiques ou lexicaux (cf. Riedel 2012 : chap. 7.2.2). Or, la position du sujet (contrôleur) par rapport au participe passé (cible) est un facteur pertinent pour la réalisation de l'accord ${ }^{8}$.

\begin{tabular}{|l|l|l|l|l|}
\hline & $\begin{array}{l}\text { Chiffres } \\
\text { absolus }\end{array}$ & $\begin{array}{l}\text { Pourcen- } \\
\text { tage }\end{array}$ & $\begin{array}{l}\text { Pourcentage } \\
\text { valable }\end{array}$ & $\begin{array}{l}\text { Pourcentage } \\
\text { accumulé }\end{array}$ \\
\hline Sujet postverbal & 17 & 4,2 & 4,2 & 4,2 \\
\hline Sujet préverbal & 392 & 95,8 & 95,8 & 100,0 \\
\hline Total & 409 & 100,0 & 100,0 & \\
\hline
\end{tabular}

Tableau 5 : Position du sujet dans les constructions non-elliptiques avec être

Le tableau 5 montre que le sujet se trouve majoritairement, c'est-à-dire dans 95,8 \% des cas, en position préverbale. Dans 4,2\% des cas, le sujet est en position postverbale. Comme on le voit dans le tableau 6, la position du sujet par rapport au participe passé joue un rôle important dans la réalisation ou non de l'accord :

\begin{tabular}{|l|l|l|l|}
\hline & Non marqué & Marqué & Total \\
\hline Sujet postverbal & 4 & 13 & 17 \\
& $23,5 \%$ & $76,5 \%$ & $100,0 \%$ \\
\hline Sujet préverbal & 28 & 364 & 392 \\
& $7,1 \%$ & $92,9 \%$ & $100,0 \%$ \\
\hline \multirow{2}{*}{ Total } & 32 & 377 & 409 \\
& $7,8 \%$ & $92,2 \%$ & $100,0 \%$ \\
\hline
\end{tabular}

Tableau 6 : Marquage de l'accord du participe passé en fonction de la position du sujet dans les constructions non-elliptiques avec être

En effet, le taux de non-accord se trouve à 23,5\% dans le cas de sujets postverbaux. Un contrôleur qui suit sa cible semble provoquer plus de cas de nonréalisation d'accord qu'un contrôleur la précédant. Or, il faut prendre en considération que le nombre des sujets postverbaux dans nos SMS est assez bas. Le

8 Dans ce qui suit nous n'avons pas pris en considération les structures elliptiques avec être, dans lesquelles le sujet, réalisé ou non, se trouve toujours en position préverbale. 
tableau indique donc tout au plus une tendance, tendance illustrée dans l'exemple suivant :

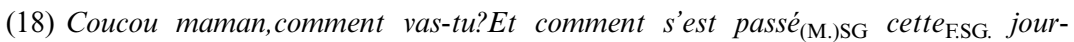
née? Est-ce que $\mathrm{tu}_{(\mathrm{F}) \mathrm{SG} .}$ es finalement quand meme allée $e_{\mathrm{F} . \mathrm{SG}}$ chez Sheila??J'espère ...Bisous, bonne soirée

L'accord n'est pas marqué ici sur le participe passé passé suivi du sujet postverbal cette journée, contrairement au participe passé allée, où la valeur [féminin] du trait [genre] du contrôleur précédant $t u$ est réalisée. Ceci indique de nouveau des raisons structurales et non pas situationnelles favorisant ou inhibant la réalisation de l'accord du participe passé.

\subsection{Le rôle des traits de l'accord}

Dans ce sous-chapitre, nous étudierons le rôle des traits dans la réalisation de l'accord dans le corpus. Comme on peut l'observer dans les deux tableaux suivants, le genre est marqué un peu moins fréquemment que le nombre, en dépit du fait que seul le genre peut avoir une réalisation phonique (" audible ») avec certains participes passés (voir section 1) :

\begin{tabular}{|l|l|l|l|l|}
\hline & $\begin{array}{l}\text { Chiffres } \\
\text { absolus }\end{array}$ & $\begin{array}{l}\text { Pourcen- } \\
\text { tage }\end{array}$ & $\begin{array}{l}\text { Pourcentage } \\
\text { valable }\end{array}$ & $\begin{array}{l}\text { Pourcentage } \\
\text { accumulé }\end{array}$ \\
\hline Non marqué & 57 & 8,0 & 8,0 & 8,0 \\
\hline Marqué & 653 & 92,0 & 92,0 & 100,0 \\
\hline Total & 710 & 100,0 & 100,0 & \\
\hline
\end{tabular}

Tableau 7 : Marquage de l'accord du participe passé selon le genre du contrôleur

\begin{tabular}{|l|l|l|l|l|}
\hline & $\begin{array}{l}\text { Chiffres } \\
\text { absolus }\end{array}$ & $\begin{array}{l}\text { Pourcen- } \\
\text { tage }\end{array}$ & $\begin{array}{l}\text { Pourcentage } \\
\text { valable }\end{array}$ & $\begin{array}{l}\text { Pourcentage } \\
\text { accumulé }\end{array}$ \\
\hline Non marqué & 18 & 2,5 & 2,5 & 2,5 \\
\hline Marqué & 692 & 97,5 & 97,5 & 100,0 \\
\hline Total & 710 & 100,0 & 100,0 & \\
\hline
\end{tabular}

Tableau 8 : Marquage de l'accord du participe passé selon le nombre du contrôleur

Tandis que le genre n'est pas marqué sur le participe passé dans $8,0 \%$ des cas, le nombre ne l'est pas dans seulement 2,5\% des cas, avec une différence d'environ $5 \%$, ce qui, avec 0,000 statistiquement, est signifiant dans le test du chi- 2 . Cette

9 Avec l'absence de marquage du nombre dans seulement 0,5\% des cas dans les constructions elliptiques avec être. Rappelons que le nombre représente un trait morphosyntaxi- 
tendance se reflète aussi dans les calculs séparés pour l'auxiliaire être ainsi que les structures elliptiques avec être (cf. Riedel 2012 : chap. 7.3.3 et 7.3.4), et pour l'auxiliaire avoir :

\begin{tabular}{|l|l|l|l|l|}
\hline & $\begin{array}{l}\text { Chiffres } \\
\text { absolus }\end{array}$ & $\begin{array}{l}\text { Pourcen- } \\
\text { tage }\end{array}$ & $\begin{array}{l}\text { Pourcentage } \\
\text { valable }\end{array}$ & $\begin{array}{l}\text { Pourcentage } \\
\text { accumulé }\end{array}$ \\
\hline Non marqué & 17 & 15,9 & 15,9 & 15,9 \\
\hline Marqué & 90 & 84,1 & 84,1 & 100,0 \\
\hline Total & 107 & 100,0 & 100,0 & \\
\hline
\end{tabular}

Tableau 9: Marquage de l'accord du participe passé selon le genre du contrôleur avec avoir

\begin{tabular}{|l|l|l|l|l|}
\hline & $\begin{array}{l}\text { Chiffres } \\
\text { absolus }\end{array}$ & $\begin{array}{l}\text { Pourcen- } \\
\text { tage }\end{array}$ & $\begin{array}{l}\text { Pourcentage } \\
\text { valable }\end{array}$ & $\begin{array}{l}\text { Pourcentage } \\
\text { accumulé }\end{array}$ \\
\hline Non marqué & 11 & 10,3 & 10,3 & 10,3 \\
\hline Marqué & 96 & 89,7 & 89,7 & 100,0 \\
\hline Total & 107 & 100,0 & 100,0 & \\
\hline
\end{tabular}

Tableau 10 : Marquage de l'accord du participe passé selon le nombre du contrôleur avec avoir

\subsection{L' « audibilité » de l'accord}

Comme esquissé dans la section 1, le fait d'avoir une réalisation phonique possible peut être considéré comme un facteur pertinent pour le marquage graphique de l'accord du participe passé, même si cette réalisation phonique ne concerne que quelques participes passés d'une fréquence pourtant considérable en français contemporain. Nous avons testé cette hypothèse en rapport avec nos données. Le tableau suivant montre que l'accord du participe passé n'est, dans 96,5\% de tous les participes passés analysés, pas réalisable phoniquement :

que interprétable, vu que le choix volontaire entre singulier et pluriel affecte la référence d'une expression nominale et joue ainsi, contrairement au genre, un rôle dans l'interprétation sémantique du nominal ou de la phrase entière. Le marquage explicite du nombre sur le participe est donc essentiel dans les constructions elliptiques pour reconstituer de façon univoque le sujet ou l'auxiliaire manquant, et par là, la signification de la construction elliptique entière. 


\begin{tabular}{|l|l|l|l|l|}
\hline & $\begin{array}{l}\text { Chiffres } \\
\text { absolus }\end{array}$ & $\begin{array}{l}\text { Pourcen- } \\
\text { tage }\end{array}$ & $\begin{array}{l}\text { Pourcentage } \\
\text { valable }\end{array}$ & $\begin{array}{l}\text { Pourcentage } \\
\text { accumulé }\end{array}$ \\
\hline Seulement graphique & 685 & 96,5 & 96,5 & 96,5 \\
\hline Réalisation phonique possible & 25 & 3,5 & 3,5 & 100,0 \\
\hline Total & 710 & 100,0 & 100,0 & \\
\hline
\end{tabular}

Tableau 11 : Réalisation phonique possible / seulement graphique de l'accord du participe passé

La pertinence du facteur « audibilité » pour le marquage graphique de l'accord ne peut pourtant pas être confirmée pour nos données :

\begin{tabular}{|l|l|l|l|}
\hline & Non marqué & Marqué & Total \\
\hline Seulement graphique & 69 & 616 & 685 \\
& 10,1 & 89,8 & 100,0 \\
\hline Réalisation phonique possible & 3 & 22 & 25 \\
& 12,0 & 88,0 & 100,0 \\
\hline \multirow{2}{*}{ Total } & 72 & 638 & 710 \\
& 0,1 & 89,9 & 100,0 \\
\hline
\end{tabular}

Tableau 12 : Marquage de l'accord du participe passé en fonction de l' « audibilité »

Les pourcentages d'accords non-marqués sont au contraire assez proches, avec $10,1 \%$ de participes passés sans réalisation phonique possible de l'accord, et $12,0 \%$ de réalisation phonique possible. Les chiffres absolus étant très bas, surtout pour le dernier groupe, et le test du chi-2 n'étant de ce fait pas permis, ce résultat n'est pas signifiant statistiquement, mais l'analyse montre tout de même une tendance qui va à l'encontre des expectations.

Pour illustrer ce paramètre d'analyse, regardons les trois exemples suivants (le premier, (17) discuté dans la section 4.2.2., est repris ici comme exemple (19)) :

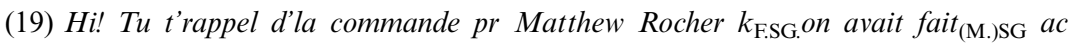
maman? Tu $l_{\mathrm{FSG}}$ 'avais mise ${ }_{\mathrm{FSG}}$ à la poste? [...].

(20) Un chouilla de retard. Y avait encore la vaisselle et Steph voulait pas partir sans $l$ F.SG'avoir faite $_{\mathrm{FSG}} \ldots ; 0$ ).

(21) Moi je $_{\mathrm{FSG}}$, me suis mise $\mathrm{F}_{\mathrm{FSG}}$ à la tache d'ouverture du courrier $[\ldots]$.

Le participe passé mise est marqué de façon correcte dans (19) et (21), avec chaque fois un clitique (objet dans (19), sujet dans (21)) précédant le participe passé comme contrôleur, le genre étant repérable uniquement grâce au cotexte précédent dans (19), et à la situation de communication (facteur externe : sexe de l'envoyeur du SMS) dans (21). La construction ne semble pas jouer de rôle ici (avec avoir dans (19) et être dans (21)). Contrairement à cela, l'accord sur fait dans (19) 
n'est pas marqué, le contrôleur étant un pronom relatif, facteur déjà identifié comme inhibant l'accord du participe passé dans les constructions avec avoir (cf. section 4.2.1.). Dans l'exemple (20), avec un objet clitisé précédant le participe passé fait et la valeur [féminin] du trait d'accord [genre], on observe pourtant une réalisation graphique de l'accord. La différence stylistique sensible dans le choix lexical entre l'exemple (21) et les deux autres ne joue du reste aucun rôle significatif en faveur ou non de l'accord du participe passé dans nos données.

\subsection{Les facteurs extralinguistiques sexe, éducation, âge}

Nous analysons dans ce qui suit l'impact des facteurs extralinguistiques sur le marquage de l'accord du participe passé. Nous avons d'abord regardé le nombre de SMS écrits par chaque participant et avons établi une moyenne de taux de marquage de l'accord pour chacun des participants. La moyenne ainsi déterminée se situe entre 0 et 1 . Dans notre évaluation, 0 représente l'accord non-réalisé, et 1 , l'accord marqué. Chaque participant possède ainsi une moyenne de marquage de l'accord. Les évaluations suivantes sont basées sur ces moyennes.

Le tableau ci-dessous représente le taux de marquage de l'accord en rapport avec le sexe des participants. Nous avons d'abord établi la moyenne de taux de marquage de l'accord chez les femmes et chez les hommes. Les femmes, qui représentent $71,7 \%$ des participants, ont un taux de réalisation de l'accord de 87,01\%, alors que le taux de marquage des hommes est, avec $95,43 \%$, plus élevé. Le taux de non-réalisation de l'accord est de $12,99 \%$ chez les femmes, et de $4,57 \%$ chez les hommes. Autrement dit, le taux de non-réalisation de l'accord chez les hommes est à peu près la moitié de celui qu'on a calculé chez les femmes. D'après l'analyse des variances ANOVA, la différence, de 0,023, est statistiquement signifiante.

\begin{tabular}{|l|l|l|l|l|}
\hline & $\begin{array}{l}\text { Chiffres } \\
\text { absolus }\end{array}$ & Moyenne & $\begin{array}{l}\text { Pourcentage accord } \\
\text { marqué }\end{array}$ & $\begin{array}{l}\text { Pourcentage accord } \\
\text { non-marqué }\end{array}$ \\
\hline Non marqué & 129 & 0,8701 & 87,01 & 12,99 \\
\hline Marqué & 51 & 0,9543 & 95,43 & 4,57 \\
\hline Total & 180 & 0,8939 & 89,39 & $10,61^{10}$ \\
\hline
\end{tabular}

Tableau 13 : Marquage de l'accord sous considération du sexe des participants

Puisque les femmes doivent effectuer plus d'accords que les hommes, p. ex. quand elles prononcent des phrases avec être dont elles sont le sujet, ce décalage s'explique, du moins partiellement.

En étudiant les tableaux 14 et 15 ci-dessous, il est évident que les participants possédant la «maturité » (= le baccalauréat) ou étant en train de la passer ont un

10 Rappelons le chapitre 4.1., où nous avons découvert que l'accord est marqué dans 89,9\% et non-marqué dans $10,1 \%$ des SMS analysés. L'aberrance minimale ici (dans les tableaux 13-16) est due à des erreurs d'arrondi, vu que les taux de marquage de l'accord des participants ont été arrondis à deux décimales. 
taux de marquage de l'accord nettement plus élevé que ceux qui ne la possèdent pas. Ils représentent dans notre corpus $87,2 \%$ de tous les participants, ce qui peut expliquer partiellement le haut pourcentage d'accord du participe passé selon la norme (presque $90 \%$ ). Le taux de non-marquage des personnes ayant le baccalauréat (ou la maturité, selon la terminologie suisse) s'élève à seulement 8,91\%. Cette observation vaut également pour les étudiants ou les personnes ayant terminé leurs études (non-marquage de 7,86\%, contre 15,29\% chez les personnes n'en ayant pas fait). Par conséquent, le taux de non-marquage de l'accord chez les participants sans maturité et sans études est à peu près deux fois plus élevé que celui des participants avec maturité et / ou enseignement supérieur. Tenant compte de ces deux facteurs, nous pouvons donc conclure que le degré d'éducation a effectivement une influence sur le marquage de l'accord. Pour ce qui est du facteur maturité, la différence dans la réalisation de l'accord est de plus statistiquement signifiante. D'après l'analyse des variances ANOVA, la signifiance est de 0,025.

\begin{tabular}{|l|l|l|l|l|}
\hline & $\begin{array}{l}\text { Chiffres } \\
\text { absolus }\end{array}$ & Moyenne & $\begin{array}{l}\text { Pourcentage accord } \\
\text { marqué }\end{array}$ & $\begin{array}{l}\text { Pourcentage accord } \\
\text { non-marqué }\end{array}$ \\
\hline Sans indication & 2 & 0,8650 & 86,50 & 13,50 \\
\hline Pas de maturité & 21 & 0,7700 & 77,00 & 23,00 \\
\hline Maturité & 157 & 0,9109 & 91,09 & 8,91 \\
\hline Total & 180 & 0,8939 & 89,39 & 10,61 \\
\hline
\end{tabular}

Tableau 14 : Marquage de l'accord sous considération du niveau de l'éducation des participants : maturité / baccalauréat

\begin{tabular}{|l|l|l|l|l|}
\hline & $\begin{array}{l}\text { Chiffres } \\
\text { absolus }\end{array}$ & Moyenne & $\begin{array}{l}\text { Pourcentage accord } \\
\text { marqué }\end{array}$ & $\begin{array}{l}\text { Pourcentage accord } \\
\text { non-marqué }\end{array}$ \\
\hline Sans indication & 2 & 0,8650 & 86,50 & 13,50 \\
\hline Pas d'études & 65 & 0,8471 & 84,71 & 15,29 \\
\hline Études & 113 & 0,9214 & 92,14 & 7,86 \\
\hline Total & 180 & 0,8939 & 89,39 & 10,61 \\
\hline
\end{tabular}

Tableau 15 : Marquage de l'accord sous considération du niveau de l'éducation des participants : études académiques

Enfin, le facteur « âge » ne s'est pas avéré pertinent pour le marquage de l'accord du participe passé dans notre corpus. Le taux est plus bas chez les jeunes participants. La moyenne de taux de non-réalisation de l'accord chez les jeunes entre 0 et 18 ans est de 13,52\%. Entre 19 et 22 ans, la moyenne est de 12,68\%. Les participants âgés de 51 à 60 ans ont avec $25 \%$ une moyenne de taux de non-marquage de l'accord plus élevée, mais celle-ci est à ignorer, car il n'y a que 6 participants dans ce groupe d'âge. Le tableau suivant révèle que l'âge n'a pas d'influence statistiquement significative sur le marquage de l'accord : 


\begin{tabular}{|l|l|l|l|l|}
\hline & $\begin{array}{l}\text { Chiffres } \\
\text { absolus }\end{array}$ & Moyenne & $\begin{array}{l}\text { Pourcentage accord } \\
\text { marqué }\end{array}$ & $\begin{array}{l}\text { Pourcentage accord } \\
\text { non-marqué }\end{array}$ \\
\hline $0-18$ & 27 & 0,8648 & 86,48 & 13,52 \\
\hline $19-22$ & 59 & 0,8732 & 87,32 & 12,68 \\
\hline $23-25$ & 37 & 0,8978 & 89,78 & 10,22 \\
\hline $26-30$ & 24 & 0,9421 & 94,21 & 5,79 \\
\hline $31-35$ & 4 & 0,9575 & 95,75 & 4,25 \\
\hline $36-40$ & 8 & 0,8963 & 89,63 & 10,37 \\
\hline $41-50$ & 12 & 0,9758 & 97,58 & 2,42 \\
\hline $51-60$ & 6 & 0,7500 & 75,00 & 25,00 \\
\hline $61-70$ & 2 & 1,0000 & 100,00 & 0,00 \\
\hline Total & 17911 & 8934 & 89,34 & 10,66 \\
\hline
\end{tabular}

Tableau 16 : Marquage de l'accord sous considération de l'âge des participants

\section{Discussion}

L'analyse quantitative de nos données, présentée en détail dans le chapitre précédent, nous a permis de constater trois faits importants.

Premièrement, le « degré de déviance », de l'orthographe du français standard contemporain est minime dans nos données (cf. tableau 1), et ceci en dépit du fait qu'il s'agit, avec les SMS ou « textos », d'une forme de communication graphique autorisant les conditions et stratégies de verbalisation de l'immédiat (quoi qu'elle ne soit pas restreinte à ces conditions et stratégies). Le projet international sms4science (cf. http://www.sms4science.org) comprend actuellement quatre autres régions de la francophonie dans lesquelles une collecte de SMS a déjà été effectuée : la Belgique, la Réunion, le Québec et la France. Une comparaison superficielle de ces différents corpus nous permet de constater le caractère particulièrement conservateur du corpus suisse, pour ce qui est de sa partie francophone, ce qui peut être mis en relation directe avec le taux élevé de participants ayant bénéficié d'une formation secondaire et académique (cf. tableaux 14 et 15). De futures recherches comparatives qualitatives et quantitatives sur les différents corpus francophones du projet sms4science qui prennent en compte ce facteur extralinguistique permettront d'éclaircir le rôle et le statut de formation des participants et de calculer le taux de réalisations graphiques « correctes » de l'accord du participe passé, indépendamment de ce facteur.

Deuxièmement, nous avons analysé deux groupes de facteurs exerçant une certaine influence sur la réalisation de l'accord du participe passé dans notre corpus :

11 Un participant n'a pas indiqué son âge. 
d'une part les facteurs linguistiques contrôlant l'accord (cf. les tableaux 2 à 10), plus le facteur « audibilité » concernant la relation phonie-graphie (tableaux 11 et 12) ; d'autre part, deux facteurs externes, le sexe et la formation des participants (cf. les tableaux 13 à 15), l'âge ne jouant très probablement aucun rôle (cf. tableau 16). Les résultats statistiques obtenus ne permettent pas d'évaluer le degré d'impact relatif de ces facteurs sur l'accord du participe passé. L'extralinguistique et le linguistique semblent constituer des groupes de facteurs pertinents, mais non hiérarchisables ou corrélables entre eux. Force est de constater que, même si le niveau d'éducation des participants s'avère statistiquement significatif pour l'accord, il n'est pas à considérer comme variable stylistique, diaphasique ou situationnelle (cf. l'introduction, avec les travaux de Söll / Hausmann ${ }^{3} 1985$ et Koch / Oesterreicher 22011). Face à ce fait, la réalisation ou non de l'accord du participe passé s'avère, du moins dans nos données, plutôt comme variable sociolinguistique, diastratique. La solution à ce problème de classification de la variation autour de l'accord du participe passé réside, bien sûr, dans la prise en compte catégorique du médium : nos données sont de nature graphique, et la réalisation de l'accord du participe passé dans la graphie n'est pas acquise naturellement, mais apprise à l'école (cf. Béguelin 2002), ce qui justifie nos observations et nous rappelle l'importance du médium dans chaque discussion ayant trait à la variation. Si la réalisation de l'accord du participe passé dans le code phonique peut être considérée comme variable stylistique, cela n'est pas le cas dans le code graphique, du moins pas d'après nos données.

Troisièmement, enfin, nous avons pu constater, pour la première fois à notre connaissance, de façon systématique et quantifiable, que les facteurs linguistiques proprement dits sont pertinents au même titre que les facteurs extralinguistiques, notamment les différents aspects de l'accord suivant la description typologique de Corbett 2006 (contrôleur, cible, traits et configuration syntaxique). Le facteur " semi-linguistique » qui concerne la relation entre phonie et graphie (marquage graphique de l'accord d'un participe passé « audible » ou non, cf. les tableaux 11 et 12), et qui est mentionné dans la littérature spécialisée (cf. p. ex. Hunnius 1980, Gutwin 1996), n'a pas d'influence sur la réalisation de l'accord dans nos données. Celles-ci confirment au contraire, de façon convaincante, des observations faites dans la recherche typologique et théorique. C'est ainsi que nous avons observé que la distance structurale relative entre la position canonique ou de base de l'argument interne du verbe lexical et sa position actuelle (surtout comme pronom relatif avec un déplacement considérable de l'argument interne en passant par le sujet de la phrase relative) est un facteur décisif pour l'absence ou le marquage déviant de l'accord du participe passé (cf. les tableaux 3 et 4). C'est donc la configuration syntaxique sous-jacente ou la différence entre celle-ci et la configuration syntaxique actuelle qui joue le rôle clé dans ces cas-là ; la nature du contrôleur (pronom relatif ou un autre élément, cf. tableau 3) n'est finalement qu'un épiphénomène. Ce sont des principes syntaxiques de localité qui permettent d'expliquer ces résultats, tout comme le fait l'auxiliaire avoir qui induit plus de cas de non-réalisation de l'accord, probablement du fait de la position marquée du contrôleur de cet accord (cf. tableau 2, contrairement à l'accord avec le sujet dans les constructions avec être). 
Deux résultats sont d'après nous les plus importants du point de vue de la syntaxe théorique. D'une part, l'observation qu'un contrôleur qui suit sa cible se trouve en corrélation avec un accord partiel ou défectif (cf. les tableaux 5 et 6 pour les sujets postverbaux avec auxiliaire être) est en parallèle avec les régularités découvertes par Greenberg 1963, ainsi qu'avec les prédictions de l'opération AGREE dans les travaux de Chomsky 2001 (cf. aussi Stark 2011 pour des observations identiques pour l'accord sujet-verbe conjugué dans le corpus suisse de SMS). D'autre part, la pertinence de l'opposition genre vs. nombre dans les traits d'accord du participe passé (cf. les tableaux 7 à 10) pour la non-réalisation de l'accord (significativement plus fréquente pour le premier), qui se trouve en accord complet avec la classification générative des traits morphosyntaxiques des langues naturelles en traits ininterprétables, purement linguistiques (comme le genre), et en traits interprétables, pertinents pour l'interprétation sémantique des phrases (cf. Radford 2004 : 287). Ceci revient à dire que toutes les « déviances » du marquage de l'accord du participe passé observées dans notre corpus et liées à des facteurs internes sont explicables sur la base de principes (morpho-) syntaxiques généraux et non pas propres au français, et semblent indépendantes du rapport notoirement difficile dans cette langue entre phonie et représentation graphique.

\section{Conclusion}

A la recherche d'une interprétation de l'absence d'accord du participe passé en français contemporain, comme simple " erreur », due pourtant à des régularités linguistiques, ou comme variable linguistique (marqueur ou indicateur variationnel), nous avons d'abord donné une brève introduction à la problématique générale et à sa description et son évaluation dans la littérature spécialisée dans le chapitre 1. Le chapitre 2 a donné un aperçu sur les éléments et mécanismes morphosyntaxiques contribuant au phénomène d'accord du participe passé dans les langues romanes et le français contemporain. Nous avons ensuite introduit dans le chapitre 3 notre base de données, à savoir la part francophone du nouveau corpus suisse de SMS, en même temps que nos facteurs d'analyse. Le chapitre 4 a résumé en détail les résultats de notre recherche quantitative, résultats qui ont été interprétés ensuite dans le chapitre 5 et ont, d'une part, mis en question le statut de l'accord du participe passé comme variable stylistique (du « français de l'immédiat »), et, d'autre part, ont démontré, pour la première fois, la haute pertinence de facteurs purement linguistiques pour la (non-)réalisation de l'accord du participe passé dans le code graphique.

Nous aimerions conclure sur une remarque méthodologique : Notre analyse a démontré la nécessité de recherches empiriques quantitatives pour pouvoir identifier l'ampleur du phénomène ainsi que sa corrélation avec différents groupes de facteurs. La discussion de données non-standard comme les SMS, exige, aussi et surtout, une approche basée sur les résultats de la recherche linguistique empirique et théorique actuelle, pour comprendre les « fautes » et la variation, et distinguer les variantes dues aux facteurs extralinguistiques, donc susceptibles de représenter 
de vraies variables sociolinguistiques ou variationnelles, des cas « mitigés » comme notre (non-)accord du participe passé, qui semble déclenché partiellement par l'externe, partiellement par l'interne, alors que finalement ces deux phénomènes sont dus aux régularités internes, purement grammaticales.

Zürich, im August 2012

\section{Bibliographie}

http://www.sms4science.ch

http://www.sms4science.org

Anis, Jacques (2007) : « Neography - Unconventional Spelling in French SMS Text Messages ", dans : Brenda Danet / Susan C. Herring (eds.), The Multilingual InternetLanguage, Culture and Communication Online, Oxford / New York : OUP, 87-115.

Auer, Peter (2000) : " On line-Syntax - oder: was es bedeuten könnte, die Zeitlichkeit der mündlichen Sprache ernst zu nehmen », dans : Sprache und Literatur 85, 43-56.

Béguelin, Marie-José (2002) : "Faut-il simplifier les règles d'accord du participe passé ? Approches interlinguistiques de la complémentation verbale : quels savoirs pour l'enseignant ? Quels savoirs pour l'élève ?", dans : Travaux neuchâtelois de linguistique (TRANEL) 37, 163-189.

Catach, Nina (102011): L'orthographe, Paris : PUF.

Chomsky, Noam (2001) : «Derivation by Phase », dans : Michael Kenstowicz (ed.), Ken Hale. A Life in Language, Cambridge/Mass. : MIT Press, 1-52.

Corbett, Greville (2006) : Agreement, Cambridge : CUP.

Dufter, Andreas / Stark, Elisabeth (2002) : « La variété des variétés : combien de dimensions pour la description?», dans : RJb 53, 81-107.

Greenberg, Joseph (1963) : "Some Universals of Grammar with Particular Reference to the Order of Meaningful Elements », dans : id. (ed.), Universals of Language, Cambridge : The MIT Press, 58-90.

Gutwin, Stefan (1996) : Der accord du participe passé als Testfall der französischen Grammatikschreibung, Tübingen : Niemeyer.

Halliday, Michael A. (1978) : Language as social semiotic: the social interpretation of language and meaning, London : Arnold.

Hunnius, Klaus (1980) : «Sprachnorm und Sprachgebrauch: Zur Geschichte der Kongruenzregeln des participe passé », dans : Hans-Dieter Bork / Artur Greive / Detlev Woll (eds.), Romanica Europaea et Americana. Festschrift für Harri Meier zum 75. Geburtstag, Bonn : Bouvier, 131-169.

Jones, Michael Allen (1996) : Foundations of French Syntax, Cambridge : CUP.

Koch, Peter / Oesterreicher, Wulf (2001) : «Gesprochene Sprache und geschriebene Sprache I Langage parlé et langage écrit », dans : Günter Holtus / Michael Metzeltin / Christian Schmitt (eds.), Lexikon der Romanistischen Linguistik, vol. I/2, Tübingen : Niemeyer, 584-627.

Koch, Peter / Oesterreicher, Wulf (2011 [1990]) : Gesprochene Sprache in der Romania: Französisch, Italienisch, Spanisch, Berlin / New York : De Gruyter.

Laenzlinger, Christopher (2003) : Initiation à la Syntaxe formelle du français : le modèle " principes et paramètres » de la grammaire générative transformationnelle, Bern : Lang. 
Lois, Ximena (1990) : " Auxiliary Selection and Past Participle Agreement in Romance », dans : Probus 2, 233-255.

Loporcaro, Michele (1998) : Sintassi comparata dell'accordo participiale romanzo, Turin : Rosenberg e Sellier.

Meisenburg, Trudel (1996) : Romanische Schriftsysteme im Vergleich: eine diachrone Studie, Tübingen : Narr.

Müller, Natascha (2000) : Ergative und unergative Verben aus romanistischer Sicht (Ms.). http://www.fb10.uni-bremen.de/iaas/workshop/ergativ/ (29/04/12)

Panckhurst, Rachel (2006) : «Le discours électronique médié : bilan et perspectives », dans : Annie Piolat (ed.), Lire, écrire, communiquer et apprendre avec Internet, Marseille : Solal, 345-366.

Perlmutter, David Michael (1978) : " Impersonal passives and the Unaccusative Hypothesis », dans : Jery J. Jaeger / Anthony C. Woodbury et al. (eds.), Proceedings of the 4th Annual Meeting of the Berkeley Linguistics Society, 18-20 Feburary 1978, Berkeley : Berkeley Linguistics Society, 157-189.

Radford, Andrew (2004) : Minimalist Syntax, Cambridge : CUP.

Remberger, Eva / Mensching, Guido (2006) : « Probes: Lack of Agreement in Romance », dans : João Costa / Maria Cristina Figueiredo (eds.), Studies on Agreement, Amsterdam : Benjamins, 173-201.

Riedel, Isabelle (2012) : L'étude de l'accord du participe passé dans les SMS suisses francophones. Unveröffentlichte Lizenziatsarbeit, Universität Zürich.

Riegel, Martin / Pellat, Jean-Christophe / Rioul, René (72009) : Grammaire méthodique du français, Paris : PUF.

Söll Ludwig / Hausmann, Franz-Josef (31985) : Gesprochenes und geschriebenes Französisch, Berlin : Schmidt.

Stark, Elisabeth (2011) : « La morphosyntaxe dans les SMS suisses francophones : Le marquage de l'accord sujet-verbe conjugué ", dans : Linguistik online 48. http://www. linguistik-online.de/48_11/stark.html

Steele, Susan (1978) : « Word order variation: a typological study », dans : Joseph H. Greenberg / Charles A. Ferguson / Edith A. Moravcsik (eds.), Universals of Human Language, vol. 4 : Syntax, Stanford : Stanford University Press, 585-623.

Tanase, Eugène (1976) : " L'accord du participe passé dans le français oral », dans : Marcel Boudreault / Frankwalt Möhren (eds.), Actes du XIII ${ }^{e}$ Congrès International de Linguistique et de Philologie Romanes, vol. 1, Québec : Presses de l'Université Laval, 475-482. 